\title{
Fundamental Parameters of Be Stars Derived from Near-Infrared Spectra
}

\author{
L. Houziaux and Y. Frémat \\ Université de Mons-Hainaut, Bro00 Mons (Belgium)
}

\begin{abstract}
Because of their spectral peculiarities, stellar parameters of objects undergoing the Be phenomenon cannot be determined in the same way as for normal B stars. In particular, the use of the first lines of the Hydrogen series is hampered by the presence of emission components. On the other end, the recently generalized use of CCD detectors, well adapted to the deep red spectral region, provides a large amount of quantitative and well calibrated flux measurements with spectral resolutions around $0.1 \mathrm{~nm}$. We propose to use the shape of the continuum and line flux distribution in the range of the higher Paschen lines of Hydrogen to determine first the effective temperature and superficial gravity of Be stars where, in most cases, the photospheric spectrum is not disturbed by line or continuum emission.
\end{abstract}

\section{Introduction}

Although the fundamental parameters (mass, radius, effective temperature, and $\mathrm{H} / \mathrm{He}$ ratio) are very similar to the $\mathrm{B}$ stars of the same subclass, their spectra may show significant differences owing to the presence of a shell or disk of circumstellar material which adds characteristic emissions both in the lines and in the continuum. Therefore, in order to have access to the general properties of the star itself, it is necessary to use spectrometric data in spectral intervals where, inasmuch as possible, the photosphere itself is likely to be seen. The wavelength ranges of interest correspond to the higher members of the series of the $H I$ spectrum because of the generally steep decrement of the line emission and the low continuum reemission (which occurs mainly at the short wavelength edge of the discontinuities). Among the series available for such determination, one has to exclude the Lyman series for several theoretical and instrumental reasons. The end of the Balmer series might be suitable, but an accurate spectrometry of this region is very often difficult to obtain because of instrumental, interstellar and atmospheric absorption. One should also be cautious in interpreting the higher Balmer line profiles because of contribution of shell lines due to the metastability of the $2 \mathrm{~s}^{2} \mathrm{~S}_{1 / 2}$ level. Finally the spectral region located between P12 and the Paschen limit seems to be the best suited spectral interval, as the interstellar and atmospheric extinction are small. Furthermore, this is a region where the response of the CCD chips is high and rather uniform, thus permitting to attain high accuracies in spectrometric measurements. 


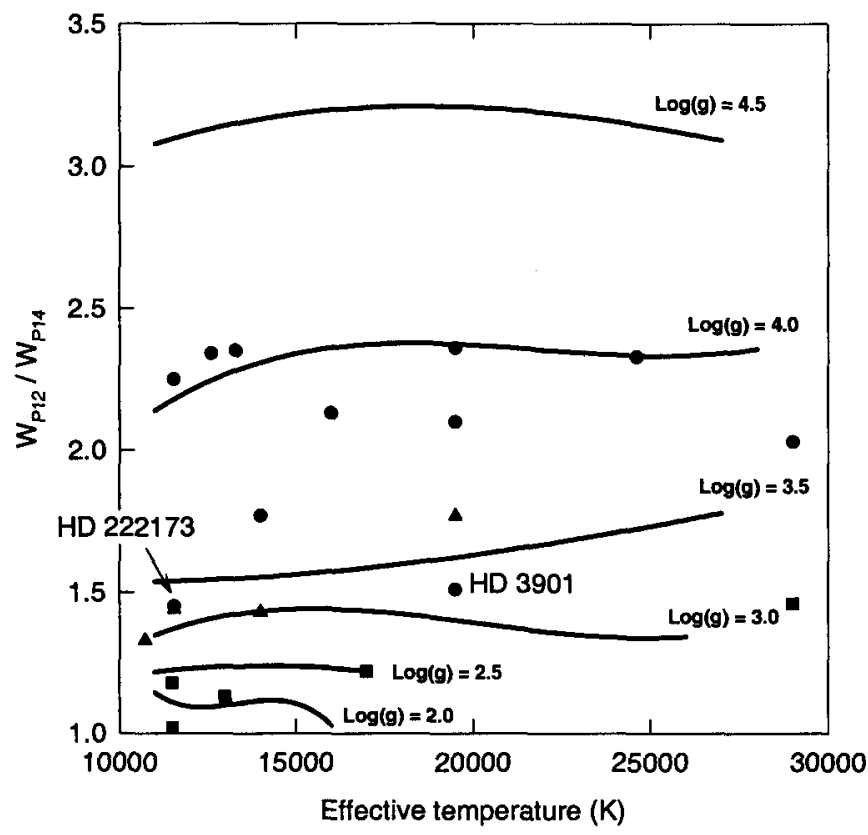

Figure 1. Solid lines indicate the computed ratio of W(P12)/W(P14) as a function of $T_{\text {eff }}$ for $\log g$ between 2.0 and $4.5 \mathrm{cgs}$. Measured values of this ratio are given for 10 class $\mathrm{V}$ stars (dots), 4 giants (triangles) and 6 supergiants (squares).

A number of $\mathrm{B}$ and $\mathrm{Be}$ stars have been observed in this deep-red region by Y. Andrillat at Observatoire de Haute-Provence (Andrillat et al. 1988, 1995) and many of these spectra are available from the CDS library. The resolution is around $0.1 \mathrm{~nm}$ and quite suitable for our purpose. In the region between 840 and $880 \mathrm{~nm}$, some members of the Paschen series are blended with lines of O I, $\mathrm{Ca}$ II and minor contributors such as He I and N I. However the lines P12 and $P 14$ are free from blending and their pseudo equivalent width may be measured with a good accuracy.

\section{Adopted procedure}

First, synthetic spectra in the range 840-880 nm have been computed in the same way as described in our previous paper (Frémat et al. 1996). We measured the pseudo-equivalent widths $\mathrm{W}(\mathrm{P} 12)$ and $\mathrm{W}(\mathrm{P} 14)$ of the unblended components $\mathrm{P} 12$ and P14. Computations have been made for effective temperatures ranging from 9000 to $35000 \mathrm{~K}$ and $\log g$ values of 2.0 to $4.5(\mathrm{cgs})$ and a solar helium abundance. Fig. 1 shows that the ratio $\mathrm{W}(\mathrm{P} 12) / \mathrm{W}(\mathrm{P} 14)$ is more sensitive to $\log g$ than to the effective temperature.

The above mentioned spectra obtained by Andrillat et al. refer to a number of MK standards and to a large number of Be stars. We corrected them for 


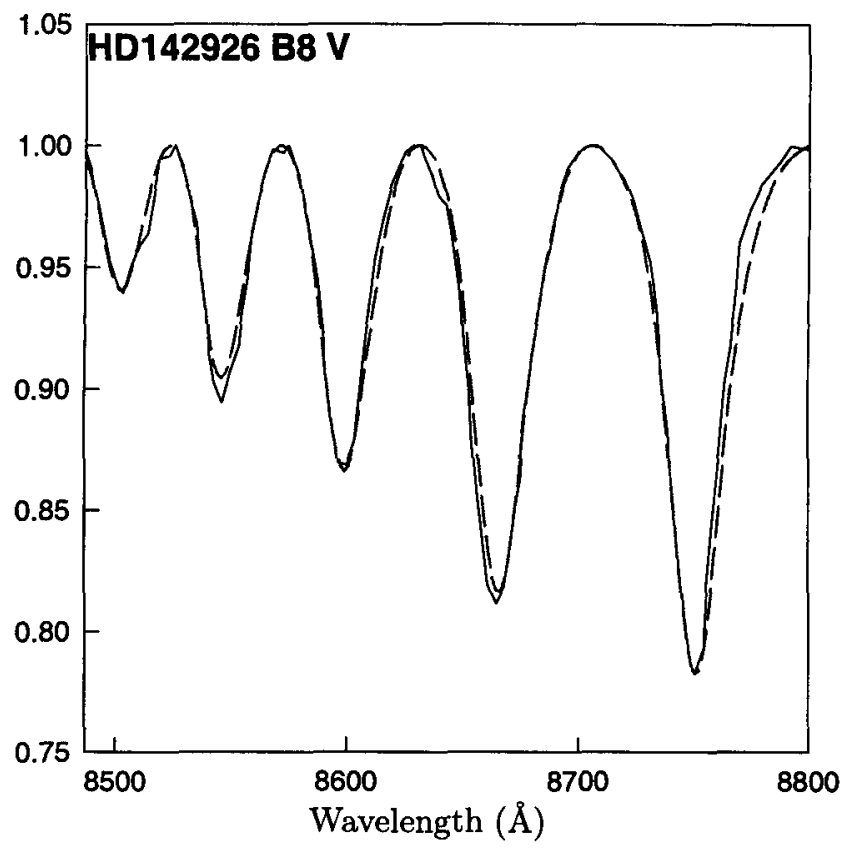

Figure 2. Comparison between the computed (dashed line) and observed (continuous line) spectra for HD142926. Fluxes are normalised relatively to the pseudo-continuum.

instrumental response in using the absolute fluxes given by Cochran and Barnes (1981) for the same MK standards. We then measured with the MIDAS software the pseudo-equivalent widths of P12 and P14 defining the pseudo-continuum at the highest flux points on either side of the line centers, and so obtained the $\mathrm{W}(\mathrm{P} 12) / \mathrm{W}(\mathrm{P} 14)$ ratio, plotted on Fig. 1, where dots refer to class V stars, triangles to giants and squares to supergiants. We also compared the observed spectra to synthetic ones to determine for the MK standards the set of $T_{\text {eff }}$ and $\log g$ that minimized the standard error and analysed the effect of the noise level on the standard error. For stars earlier than B3, the relative error on $\log g$ is not larger than $3 \%$ but the uncertainty on the effective temperature may reach $4 \%$. Furthermore, we checked that the values obtained for the "spectroscopic" gravity for standard stars were in fair agreement with "mechanical" values of the gravity deduced from masses and radii. Therefore, using Hipparcos parallaxes and monochromatic magnitudes derived from the sky-scanning experiment S2/68 aboard the TD1 satellite (Houziaux, 1992), we determined the radii in comparing the observed fluxes to the theoretical fluxes given by Kurucz (1993). From the star's luminosity and hence its location in the HR diagram, we obtained its mass among the evolutionary tracks of Schaller et al. (1992). The values obtained for the gravity were in good agreement with the "spectroscopic" gravities derived from the ratio $\mathrm{W}(\mathrm{P} 12) / \mathrm{W}(\mathrm{P} 14)$. 


\section{Results}

The method was finally applied to a sample of Be stars and the results are given in Table 1. These parameters lead to a good agreement with the synthetic and observed spectra when the appropriate projected rotational velocity of the star is taken into account, as shown in Fig. 2 for the star HD 142926. However, the observational data so far available for Be stars have a lower resolution $(\sim 0.5$ $\mathrm{nm}$ ) than the spectra of the standard stars. This affects mostly the shape of the pseudo-continuum and degrades somewhat the accuracy of the results. More precise values of the parameters require new observations of these Be stars with a resolution of about $0.1 \mathrm{~nm}$. Details on the calculations and further results will be published elsewhere.

Table 1. Fundamental parameters derived from the fitting of the Paschen series.

\begin{tabular}{rccc}
\hline \multicolumn{1}{c}{ HD } & Sp. Type & $T_{\text {eff }}$ & $\log g$ \\
\hline \hline 21650 & B5 V & 13500 & 3.78 \\
23480 & B6 IV & 14300 & 3.75 \\
45542 & B5-6 IV & 14600 & 4.00 \\
89884 & B5 V & 16300 & 4.10 \\
138749 & B5 V & 14000 & 3.62 \\
142926 & B8 V & 12000 & 3.80 \\
175863 & B5 III & 15400 & 3.45 \\
183914 & B8 V & 12800 & 3.75 \\
192044 & B7 V & 14000 & 4.08 \\
193911 & B8 III & 14600 & 3.60 \\
198183 & B5 V & 14200 & 3.90 \\
205551 & B9 III & 10900 & 3.25 \\
209409 & B6 IV & 13000 & 3.90 \\
210129 & B7 V & 13900 & 3.96 \\
\hline
\end{tabular}

Acknowledgments. L. Houziaux would like to thank the National Fund for Scientific Research (FNRS) for financial support.

\section{References}

Andrillat, Y., Jaschek, M., Jaschek, C. 1988, A\&AS 72, 129

Andrillat, Y., Jaschek, C., Jaschek, M. 1995, A\&AS 112, 475

Cochran, A., Barnes, T. 1981, ApJS 45, 73

Frémat, Y., Houziaux, L., Andrillat, Y. 1996 , MNRAS 279,25

Houziaux, L. 1992 Ultraviolet monochromatic magnitudes of Be stars from TD1 observations (unpublished)

Jaschek, C. Andrillat, Y. 1998, A\&A 331, 314

Kurucz, L., 1993, CDROM N13

Schaller, G., Schaerer, D., Meynet, G., Maeder, A. 1992, A\&AS 96, 269 